\title{
Cloning and functional analysis of the FAD2 gene family from desert shrub Artemisia sphaerocephala
}

\author{
Xiumei Miao ${ }^{\dagger}$, Lijing Zhang ${ }^{* \dagger} \mathbb{D}$, Xiaowei Hu, Shuzhen Nan, Xiaolong Chen and Hua Fu
}

\begin{abstract}
Background: Linoleic acid is an important polyunsaturated fatty acid, required for all eukaryotes. Microsomal delta$12\left(\Delta^{12}\right)$ oleate desaturase (FAD2) is a key enzyme for linoleic acid biosynthesis. Desert shrub Artemisia sphaerocephala is rich in linoleic acid, it has a large FAD2 gene family with twenty-six members. The aim of this work is to unveil the difference and potentially functionality of AsFAD2 family members.
\end{abstract}

Results: Full-length cDNAs of twenty-one AsFAD2 genes were obtained from A. sphaerocephala. The putative polypeptides encoded by AsFAD2 family genes showed a high level of sequence similarity and were relatively conserved during evolution. The motif composition was also relatively conservative. Quantitative real-time PCR analysis revealed that the AsFAD2-1 gene was strongly expressed in developing seeds, which may be closely associated with the high accumulating ability of linoleic acid in A. sphaerocephala seeds. Although different AsFAD2 family members showed diverse response to salt stress, the overall mRNA levels of the AsFAD2 family genes was stable. Transient expression of AsFAD2 genes in the Nicotiana benthamiana leaves revealed that the encoded proteins were all located in the endoplasmic reticulum. Heterologous expression in Saccharomyces cerevisiae suggested that only three AsFAD2 enzymes, AsFAD2-1, - 10, and - 23, were $\Delta^{12}$ oleate desaturases, which could convert oleic acid to linoleic acid, whereas AsFAD2-1 and AsFAD2-10 could also produce palmitolinoleic acid.

Conclusions: This research reported the cloning, expression studies, subcellular localization and functional identification of the large AsFAD2 gene family. These results should be helpful in understanding fatty acid biosynthesis in A. sphaerocephala, and has the potential to be applied in the study of plant fatty acids traits.

Keywords: Fatty acid desaturase, linoleic acid, expression analysis, subcellular localization, yeast expression

\section{Background}

Linoleic acid (LA, C18:2) is known as an important polyunsaturated fatty acid (PUFA), required for normal growth of all eukaryotes [1]. LA is a precursor for the synthesis of other PUFAs such as linolenic acid and arachidonic acid, and physiologically active regulatory compounds such as prostaglandin [2]. LA has the effect of lowering serum cholesterol and triglyceride levels, which is good for preventing cardiovascular diseases,

\footnotetext{
* Correspondence: lijingzhang501@hotmail.com; fuhua@|zu.edu.cn ${ }^{+}$Xiumei Miao and Lijing Zhang contributed equally to this work. State Key Laboratory of Grassland Agro-ecosystems; Key Laboratory of Grassland Livestock Industry Innovation; Ministry of Agriculture and Rural Affairs; Engineering Research Center of Grassland Industry, Ministry of Education; College of Pastoral Agriculture Science and Technology, Lanzhou University, Lanzhou 730020, People's Republic of China
}

such as atherosclerosis and myocardial infarction [3]. In addition, LA is also the precursor of conjugated linoleic acid (CLA), which is mainly generated in the rumen of ruminant animals and has been shown to enhance immune function and exert multiple beneficial effects in obesity, cancer, inflammatory diseases, and hypertension $[4,5]$. However, LA cannot be synthesized by humans and other mammals, and must be consumed through diet to support normal physiological metabolism [6]. In plants, higher LA content helps maintaining the fluidity and integrity of the cell membrane, which is beneficial for their adaptation to various biotic or abiotic stresses $[7,8]$.

LA synthesis in plants is usually catalyzed by membrane-bound enzymes $\Delta^{12}$ fatty acid desaturases (FADs), it is also known as $\omega-6$ FADs, which act by introducing a double bond at the delta-12 $\left(\Delta^{12}\right)$ position 
of the oleic acid carbon chain [9]. According to the location in the endoplasmic reticulum (ER) or plastids, $\Delta^{12}$ FADs are divided into microsomal (FAD2) and plastid (FAD6) enzymes. In recent years, FAD2 genes have been identified and functionally analyzed in a variety of organisms, including plants, fungi, and some other lower animals $[1,10]$. To date, the FAD2 gene has been cloned from many plant species. With the exception of Arabidopsis thaliana, which has only one FAD2 gene [11], most plants have multiple FAD2 genes. Thus, olive (Olea europaea) has two FAD2 genes [12], Oilseed rape (Brassica napus) has four [13], peanut (Arachis hypogaea) has six [14], safflower (Carthamus tinctorius) has eleven [15], and Artemisia sphaerocephala has twenty-six [16]. Gene families usually occur through gene duplication and mutations, and the variations in the number of gene family members is an important evolutionary mechanism underlying functional diversity and shaping genomic adaptation in various species [17]. Therefore, different numbers of the $F A D 2$ genes in plants may be a result of their adaptation to diverse environmental conditions.

The FAD2 enzymes play an important role in plant fatty acid synthesis, and consequently, in their growth, development, and resistance to low temperatures and high salt concentrations, as well as other biotic and abiotic stresses [8]. Thus, it was found that Arabidopsis lacking the FAD2 gene has reduced tolerance to cold [18] and increased sensitivity to salt at the seed germination and seedling stages [19]. The safflower CtFAD2-1 gene, which was expressed in developing seeds, was mostly responsible for the desaturation of storage lipids; thus, CtFAD2-3, $-4,-6$, and -7 were mainly expressed in the cotyledons and hypocotyls of seedlings, whereas CtFAD2-5 and -8 were specifically expressed in roots and CtFAD2-10 in flowers, were mostly responsible for the desaturation of membrane lipids [15]. In cotton, the expression of FAD2-3 and FAD2-4 genes were induced under cold stress, whereas that of FAD2-2 was not affected [20]. Heterologous expression of sunflower FAD2-1 and FAD2-3 genes in yeast cells resulted in the increase of dienoic fatty acid content, which give the help of enhancing the freeze and salt tolerance of yeast [21]. Two ShFAD2 genes from Salvia hispanica shared a similar expression pattern, either induction or suppression, in response to various abiotic stresses [22]. Overall, these findings indicated that different $F A D 2$ genes of the same plant may vary not only in their tissue expression patterns and functional characteristics, but also in responses to environmental stresses. At present, the research on FAD2 genes is mainly conducted in model plants and oil crops, and there is no information on the expression and functional activity of FAD2 genes from the desert plant $A$. sphaerocephala, which has the largest $F A D 2$ gene family among the investigated plants.
Artemisia sphaerocephala Kraschen, which belongs to the Artemisia genus of the Compositae family, is a perennial wild shrub widely distributed in the moving and semi-stable sand dunes in the deserts of northern China [23]. Artemisia sphaerocephala seeds contain $21.5 \%$ oil and can be used to produce biodiesel [24], nearly $90 \%$ of seeds oil are unsaturated fatty acids, especially LA, constituting over $78 \%$ of total fatty acids [25]. Comparing with other plants, such as sunflower, soybean, and peanut, A. sphaerocephala seeds and leaves can accumulate much more LA [16]. The maintenance of high degree of membrane lipid unsaturation under stress conditions is one of the important stress adaptation mechanisms in plants, previous studies showed that $A$. sphaerocephala is resistant to drought and salt by maintaining high LA content [26, 27]. Twenty-six FAD2 genes were identified in A. sphaerocephala, which is the largest FAD2 gene family reported till now [16]. In this study, we cloned full-length cDNA of the $A$. sphaerocephala FAD2 (AsFAD2) gene family members, and analyzed their structural characteristics, tissue distribution, and expression levels under high salt stress conditions. Using heterologous expression systems, we also evaluated subcellular localization and functional activity of AsFAD2 proteins. These results should be helpful in further understanding of the roles of the AsFAD2 gene family in the maintenance of high LA content in $A$. sphaerocephala.

\section{Results}

Cloning and analysis of the full-length cDNA of AsFAD2 gene family.

We cloned the full-length cDNAs of twenty-one AsFAD2 genes from different $A$. sphaerocephala tissues using reverse transcription PCR (RT-PCR) and rapid amplification of cDNA ends (RACE) methods based on transcriptome sequence data (Additional file 1: Table S1). However, because of the short lengths of the core fragments and low expression levels of these genes in the tissue the full-length cDNAs of AsFAD2-3, - 17, $18,-25$, and -26 genes were not obtained. The size of full-length cDNAs for the twenty-one AsFAD2 genes varied between 1320 and $1728 \mathrm{bp}$, whereas the length of 5' UTRs and 3' UTRs were between 27 and $373 \mathrm{bp}$ and $87-279 \mathrm{bp}$, respectively, and the predicted protein sizes were between 371 and 429 amino acids. The theoretical molecular masses and isoelectric points of predicted proteins were about 43.50-49.13 and 6.22-8.83, respectively. According to grand average of hydropathicity (GRAVY) analysis, AsFAD2-2, $-7,-14$, and -23 genes encoded hydrophobic proteins, whereas the other genes encoded hydrophilic proteins, as they had positive and negative GRAVY values differently. The predicted transmembrane number was between 3 and 6. Plant-mPLoc 
analysis predicted that the twenty-one AsFAD2 genes were located in the ER.

Sequence identity of multiple members of AsFAD2 gene family.

Sequence similarity among the coding regions of twenty-one AsFAD2 genes at the amino acid level was presented in Additional file 2: Fig. S1. The result showed that the pairwise similarity of AsFAD2-1 and AsFAD212, AsFAD2-16 and AsFAD2-19 were identical with the similarity level of $100.00 \%$, whereas there was only one amino acid different in the pairwise similarity of AsFAD2-5 and AsFAD2-16/19, AsFAD2-6 and AsFAD2-24, AsFAD2-7 and AsFAD2-14, namely, the similarity levels among these amino acid sequences were 99.74\%. Thus, the AsFAD2-1, AsFAD2-5, AsFAD2-6, and $A s F A D 2-7$ were selected for further study. The putative amino acid sequences of sixteen AsFAD2 genes were significantly different, and the similarity level range from 36.54 to $97.85 \%$.

Phylogenetic and motif analysis of encoded proteins of AsFAD2 gene family.

To elucidate phylogenetic relationship of the AsFAD2 gene family, the deduced polypeptide sequences of the selected sixteen AsFAD2 genes (Additional file 3: Table S2) were aligned with FAD2 sequences of other plants, including oil plants, model plants, and some plant with divergent FAD2 fatty acid modifying enzymes (Fig. 1). Phylogenetic analysis showed that the sixteen AsFAD2 were divided into seven groups. AsFAD2-1 was clustered with other seed expressed FAD2s, such as sunflower HaFAD2-1 and safflower CtFAD2-1. AsFAD2-10 was clustered together with other constitutively expressed FAD2s, such as sunflower HaFAD2-2, HaFAD2-3, and safflower CtFAD2-2. AsFAD2-23 was clustered together with fatty acid acetylenases and hydroxylases from other plants. AsFAD2-9 and CtFAD2-9, AsFAD2-2, - 5, - 6, 15 and $C t$ FAD2-8, and $A s F A D 2-11$ and $C t$ FAD2-7 were positioned next to each other, respectively, in the same branch. AsFAD2-4, -8 , and -21 were clustered with fatty acid conjugases from Calendula officinalis. AsFAD2-7, $13,-20$, and -22 proteins were clustered together with fatty acid acetylenases and epoxygenases from several plant species.

The alignment of putative AsFAD2 polypeptides together with selected plant orthologs was shown in Additional file 4: Fig. S2. The AsFAD2 polypeptides contained $\mathrm{C}$-terminal aromatic amino acid-rich motifs. For example, AsFAD2-1, AsFAD2-2, and AsFAD2-4 had YKNKM, FKNKL and WFKK, respectively. Additionally, AsFAD2 family proteins contained three highly conserved histidine-rich motifs. Motifs of FAD2 protein sequences of sixteen A. sphaerocephala, one Arabidopsis thaliana and one Nicotiana tabacum were analyzed (Fig. 2). The detailed information of twenty putative conserved motifs were shown in Additional file 5: Fig. S3. These proteins all had nine conserved motifs, including motif $1,2,3,4,6,7,8,9$, and 11 . The motif composition of the AsFAD2 family proteins was relatively conserved. AsFAD2-2, - 5, - 6 and -15 were clustered together to be a branch (Fig.1), and they all had fourteen identical motifs, AsFAD2-5 and AsFAD2-15 contained motif 19. AsFAD2-9 and AsFAD2-2 were next to each other, and had same motifs. The motif composition of AsFAD2-23 was different from other AsFAD2s. AsFAD2-10, AtFAD2, $A s$ FAD2-1 and NtFAD2 were clustered together to be a branch (Fig. 1). The motifs of AtFAD2, AsFAD2-1 and NtFAD2 were completely identical. AsFAD2-10 lacked motif 16. AsFAD2-4, $-8,-11$ and -21 were located next to each other and formed a branch (Fig. 1), AsFAD2-4 and $A s F A D 2-21$ had same motifs. In contrast, AsFAD2-8 contained motif 14 and lacked motif 12, AsFAD2-11 had motif 12 . AsFAD2-7, $-13,-20$ and -22 were situated next to each other and formed a branch (Fig. 1), and they all had fifteen same motifs.

Expression analysis of AsFAD2 gene family in $A$. sphaerocephala.

The transcript levels of the sixteen AsFAD2 genes in different $A$. sphaerocephala tissues were detected using quantitative real-time PCR (qRT-PCR). It displayed that the expression patterns of $A s F A D 2$ gene family were diverse, and they may play different functions roles in different tissues and organs. The numbers and relative expression levels of AsFAD2 genes increased significantly in the seed swelling and germination, especially the expression levels of $A s F A D 2-2,-15,-20$ increased significantly (Fig. 3a-c). The expression level of AsFAD2-15 was the highest in roots (Fig. 3d). AsFAD2-15 and AsFAD2-20 showed high expression levels in stems and leaves (Fig. 3d-f). In flower buds and flowers, the expression levels of AsFAD2-20 and AsFAD2-13 were the highest (Fig. 3g-h), respectively, compared to other AsFAD2s. AsFAD2-1 was strongly expressed in developing seeds, but had low expression levels in other tissues, belonging to gene of the seed-type expression. AsFAD210 was expressed in all the checked tissues, belonging to gene of the constitutive expression. AsFAD2-1 and AsFAD2-10 may play an important role in the formation of high linoleic acid in A. sphaerocephala seeds (Fig. 3i-j).

According to the expression pattern of AsFAD2 genes in various $A$. sphaerocephala organs, eleven genes with high expression in leaves were selected to analyze their response to salt stress (Fig. 4). Forty-five day-old seedlings were treated with 50 and $200 \mathrm{mM} \mathrm{NaCl}$ for 7 days, and the relative expression of the AsFAD2 genes in leaves was compared with that in untreated control plants. At $50 \mathrm{mM} \mathrm{NaCl}$, the expression of AsFAD2-1 and -10 were downregulated significantly, whereas that of AsFAD2-2, - 15, and - 22 were upregulated, and that 


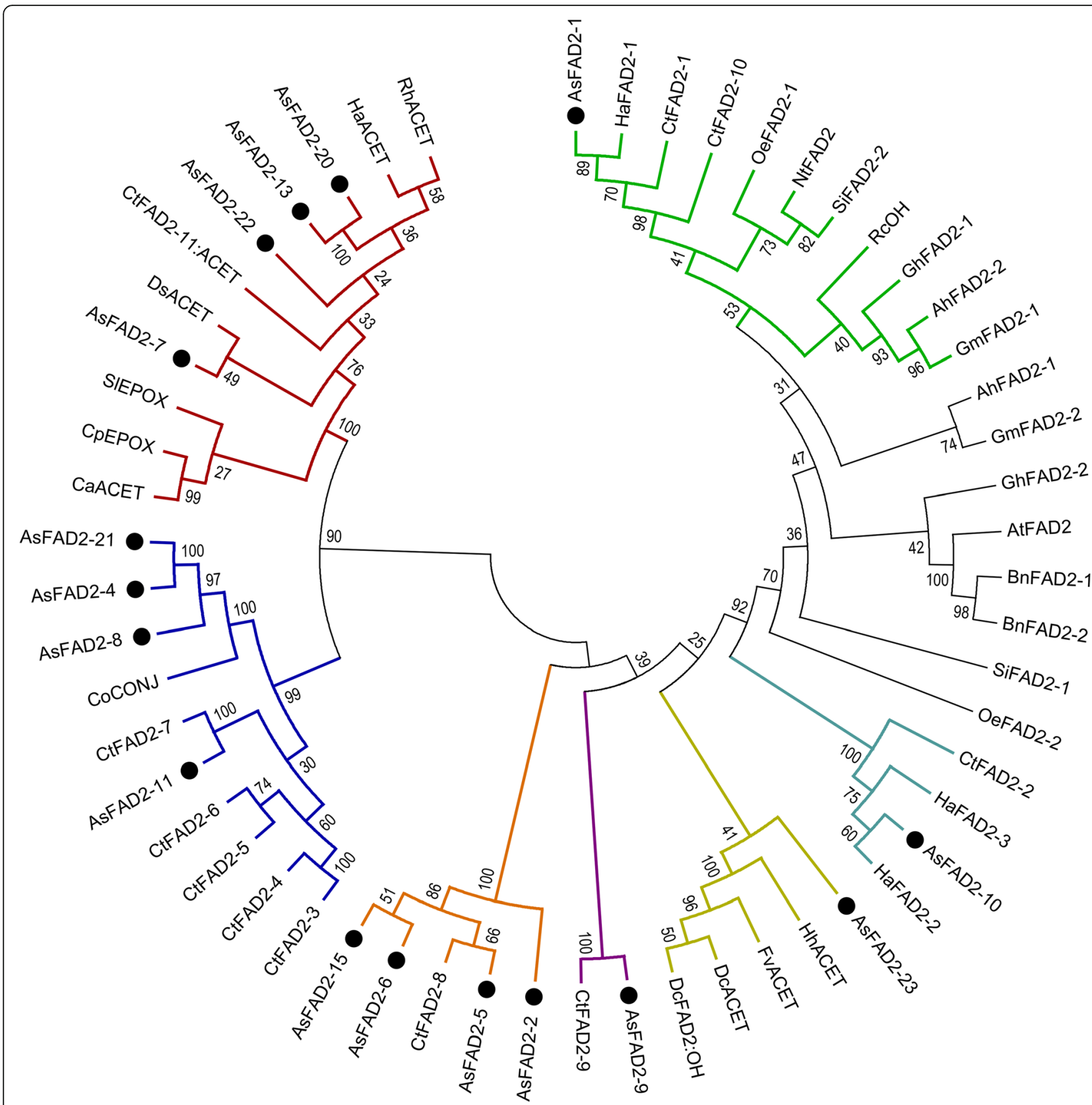

Fig. 1 Phylogenetic comparison of AsFAD2s and other plants FAD2s. The phylogenetic tree was generated by MEGA6.0. FAD2 desaturases (FAD), hydroxylases $(\mathrm{OH})$, epoxygenases (EPOX), acetylenases (ACET) and conjugases (CONJ) from various plants were included in the alignment. The GenBank accession numbers of the amino acid sequences represented in the phylogenetic tree were: AhFAD2-1, ACZ06072.1; AhFAD2-2, AHN60569.1; sixteen AsFAD2 proteins; AtFAD, AAM61113.1; BnFAD2-1, AAF78778.1; BnFAD2-2, AAS92240.1; CaACET, ABC00769.1; CoCONJ, AAK26632.1; CpEPOX, CAA76156.1; CtFAD2-1, AGC65498.1; CtFAD2-2, AGC65499.1; CtFAD2-3, AGC65500.1; CtFAD2-4, AGC65501.1; CtFAD2-5, AGC65502.1; CtFAD2-6, AGC65503.1; CtFAD2-7, AGC65504.1; CtFAD2-8, AGC65505.1; CtFAD2-9, AGC65506.1; CtFAD2-10, AGC65507.1; CtFAD211:ACET, AGC65508.1; DCACET, AAO38033.1; DcFAD2:OH, AAK30206.1; DsACET, AAO38036.1; FvACET, AAO38034.1; GhFAD2-1, CAA65744.1; GhFAD2-2, CAA71199.1; GmFAD2-1, AAB00859.1; GmFAD2-2, AAB00860.1; HaFAD2-1, AAL68981.1; HaFAD2-2, AAL68982.1; HaFAD2-3, AAL68983.1; HaACET, ABC59684.1; HhACET, AAO38031.1; NtFAD, AAT72296.2; OeFAD2-1, AAW63040.1; OeFAD2-2, AAW63041.1; RCOH, AAC49010.1; RhACET, AAO38035.1; SiFAD2-1, XP_011075145.1; SiFAD2-2, XP_011080227.1; and SIEPOX, AAR23815.1 (Ah, Arachis hypogaea; As, Artemisia sphaerocephala; At, Arabidopsis thaliana; Bn, Brassica napus; Ca, Crepis alpine; Co, Calendula officinalis; Cp, Crepis palaestina; Ct, Carthamus tinctorius; Dc, Daucus carota; Ds, Dimorphotheca sinuate; Fv, Foeniculum vulgare; Gh, Gossypium hirsutum; Gm, Glycine max; Ha, Helianthus annuus; Hh, Hedera helix; Nt, Nicotiana tabacum; Oe, Oleaeuropaea; Rc, Ricinus communis; Rh, Rudbeckia hirta; Si, Sesamum indicum; SI, Stokesia laevis) 


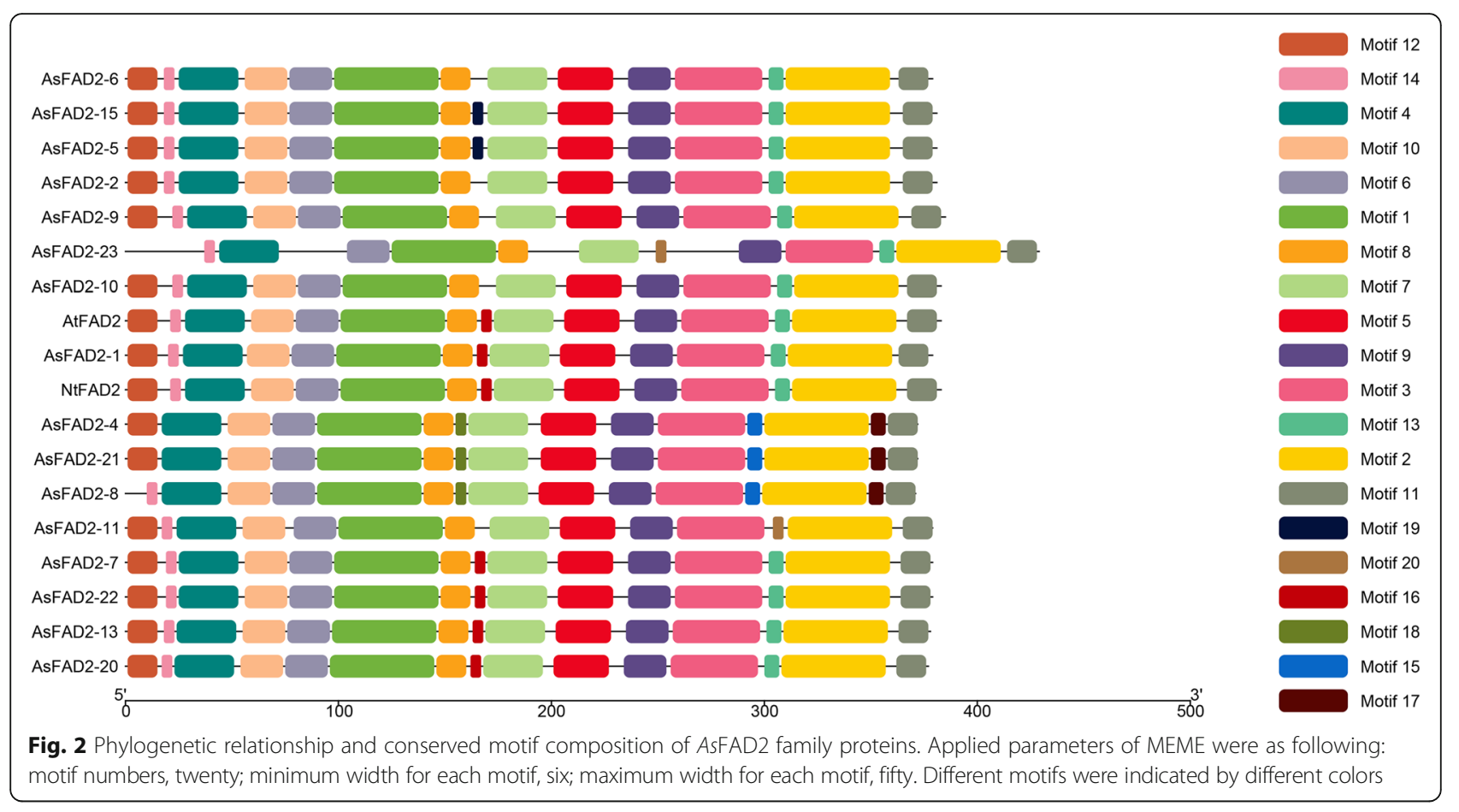

of the other genes were unchanged compared to control. At $200 \mathrm{mM} \mathrm{NaCl}$, the expression of AsFAD2-2 and - 5 genes were increased significantly, whereas that of AsFAD2-7 was decreased significantly, and that of the other genes showed no difference compared to control. Overall, mRNA expression of eleven AsFAD2 genes was not significantly changed with $\mathrm{NaCl}$ treatment.

Subcellular Localization of AsFAD2 proteins.

Based on phylogenetic relationship and tissue expression patterns, seven AsFAD2 genes were selected for subcellular localization analysis, including AsFAD2-1, $9,-10,-11,-15,-20$, and -23 . The results showed that seven AsFAD2 cDNA-encoded proteins were localized to network-like organelles, the strong green fluorescent protein (GFP) and red fluorescent protein (RFP) signals were observed in the epidermal cells of tobacco leaves, and the both fluorescent signals could be overlapped and displayed as yellow fluorescent signals, indicating that the selected seven AsFAD2s were transiently expressed in the ER of tobacco leaf epidermal cells (Fig. 5). It was speculated that the other AsFAD2 proteins could also be located in the ER.

Functional analysis of $A s F A D 2$ genes in yeast.

Sixteen AsFAD2 family members were expressed in the Saccharomyces cerevisiae INVSc1, and the fatty acid compositions of the yeasts were analyzed (Fig. 6 and Additional file 6: Table S3). The results indicated that dienoic fatty acids, including palmitolinoleic acid (C16:2) and LA (C18:2), were not produced in the yeast with the empty pYES2 vector (Fig. 6a). However, C18:2 content was respectively $18.58,16.54$ and $3.29 \%$ of total fatty acids in the transformed yeast expressing the AsFAD2-1 (Fig. 6b), AsFAD2-10 (Fig. 6c), and AsFAD2-23 (Fig. $6 \mathrm{~d})$, the conversion ratio of $\mathrm{C} 18: 1$ to $\mathrm{C} 18: 2$ were 60.07 , 57.49 and $12.78 \%$, respectively (Additional file 6: Table S3). In addition, C16:2 was detected in the transformed yeast strains expressing AsFAD2-1 and AsFAD2-10, C16:2 content was respectively 18.10 and $9.95 \%$, and the conversion ratio were 36.41 and $18.82 \%$, respectively (Additional file 6: Table S3). However, no corresponding fatty acid product was detected in yeast cells expressing other genes (Additional file 6: Table S3).

\section{Discussion}

Among plant species examined to date, A. sphaerocephala had the largest FAD2 family containing twenty-six genes, which was much more than in the next largest family in safflower (11 genes) [15]. In this study, we isolated twenty-one AsFAD2 genes from A. sphaerocephala (Additional file 1: Table S1), including sixteen AsFAD2 genes with distinct coding regions (Additional file 2: Fig. S1). The AsFAD2 family members contained uninterrupted coding region sequences, which were highly homologous and relatively conserved during evolution (Fig. 2). Similarly, in safflower, the coding regions of CtFAD2 genes did not contain introns. Therefore, the formation of the gene family was suggested to be most likely caused by gene duplication rather than nucleotide alternative splicing [15]. Whole-genome sequencing of soybean revealed two genome duplication events occurred 


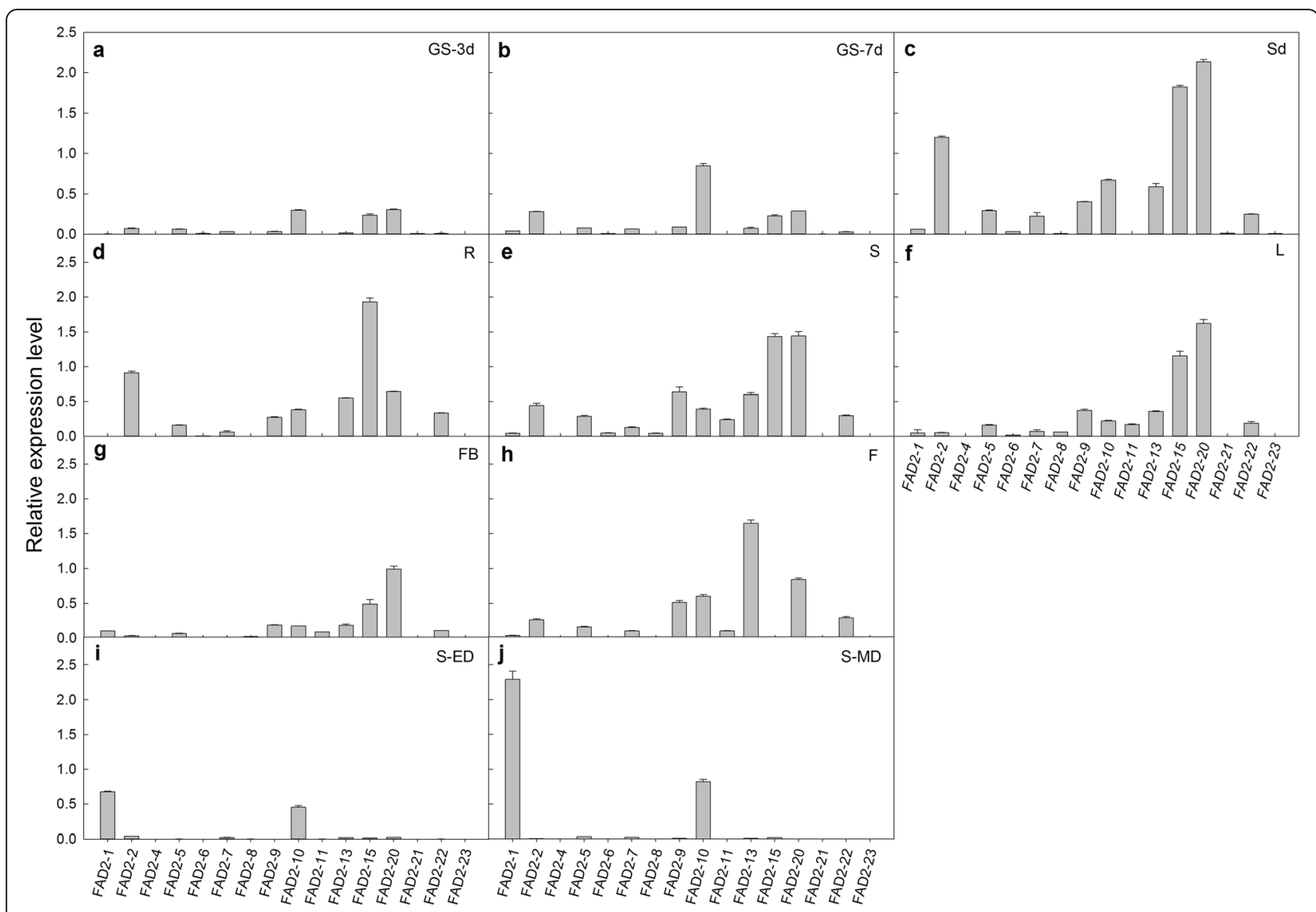

Fig. 3 Relative expression levels of sixteen AsFAD2 genes in various A. sphaerocephala tissues. a GS-3d (Germinated Seeds after 3 days). b GS-7d (Germinated Seeds after 7 days). c Sd (Seedlings). d R (Roots). e S (Stems). f L(Leaves). g FB (Flower Buds), h F (Flowers). i S-ED (Early Developing Seeds). j S-MD (Mid-Developing Seeds). The actin gene was used as an internal reference gene. The graph shows average values of three replicates with the respective error bars indicative of standard error

fifty-nine and thirteen million years ago [28], and seven soybean FAD2 genes were generated as a result in previous study [29]. In cucumber, two FAD2 genes also originated through gene duplication [30]. A. sphaerocephala is a cross-pollinated diploid wild plant [31], in the absence of genomic data, it is unclear whether this species has undergone whole-genome duplication or not. Therefore, further research was needed to determine how FAD2 gene family was emerged in A. sphaerocephala with a large quantity. However, based on the results of this study, it could be inferred that the formation of AsFAD2 gene family may be similar to that in safflower, i.e., could be a result of gene duplication.

The deduced amino acid sequences of the AsFAD2 family members contain aromatic amino acid-rich motifs at the C-terminus and three highly conserved histidinerich motifs, which had similarities as well as differences compared with those in other plants (Additional file 4: Fig. S2), indicated complexity of the AsFAD2 gene family and more possibilities for the diversification of AsFAD2 enzymes. In addition, the predicted AsFAD2 proteins contain between three and six transmembrane regions (Additional file 1: Table S1), which played an important role in FAD2 catalytic activity [32], and confirmed that the A. sphaerocephala FADs were membrane-bound. The number of transmembrane domains among plant FAD2 enzymes is different, it is usually in the range of three to six. Thus, red flax (Linum grandiflorum), pumpkin (Cucurbita pepo), sesame (S. indicum), and grape (Vitis labrusca) enzymes contain 3, 4, 5, and 6 transmembrane regions, respectively [33, 34]. Our data indicated that the AsFAD2 family members were structurally diverse.

The FAD2 enzymes not only have desaturase activity, but can also perform other fatty acid modifications, including hydroxylation [35], epoxidation [36], and formation of acetylene bonds [37, 38] and conjugated double bonds $[39,40]$. Some FAD2 enzymes had more than two functions. For example, LfFAD2 of Lesquerella fendleri was a bifunctional enzyme with dehydrogenase and hydroxylase activity [41],whereas Crepis alpina CaFAD2 and safflower CtFAD2-11 were tri-functional 


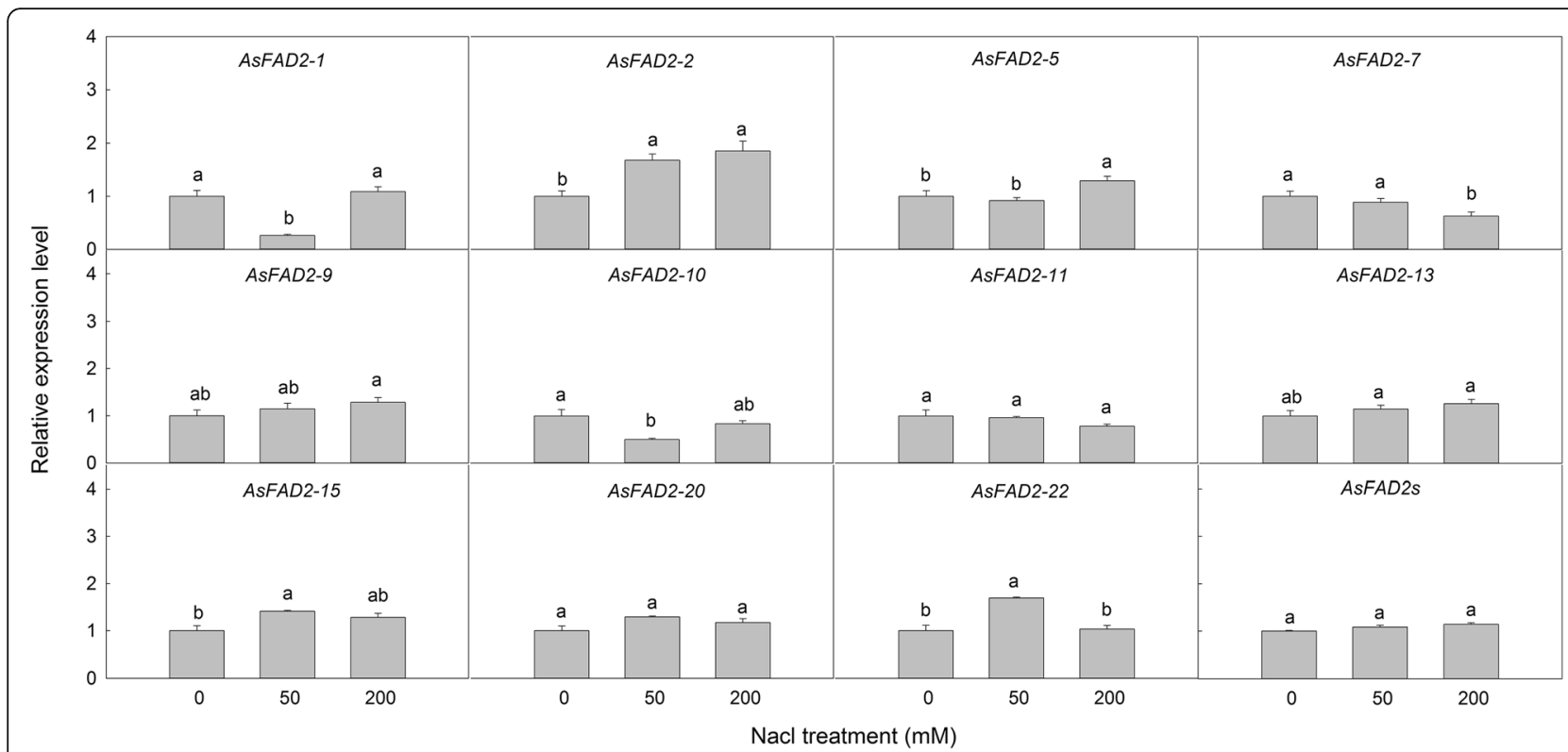

Fig. 4 Expression analysis of eleven AsFAD2 genes in A. sphaerocephala leaves treated with $\mathrm{NaCl}$. Values are means \pm SE $(n=5)$ and bars indicate SE. Different letters within a column indicated significant difference at $P<0.05$

enzymes as they can introduce a carbon double bond in either cis or trans configuration or acetylenic bond at the $\Delta 12$ position $[15,42]$. Based on phylogenetic relationship inferred AsFAD2-4, - 8, - 11, and - 21 proteins may be conjugated enzymes, AsFAD2-7, -13, - 20, and - 22 may have acetylene and cyclooxygenase activities, and AsFAD2-23 may have acetylase and hydroxylase activities (Fig. 1). However, no corresponding fatty acid products were detected in transgenic yeast cells (data not presented). These results illustrated that these AsFAD2s did not have the functions of fatty acid modifying enzymes in transgenic yeast, which was consistent with the result of safflower CtFAD2 gene family [15].

Current studies have shown that although the expression and function of $F A D 2$ genes in plants have temporal and spatial differences, roughly two types of expression patterns, constitutive and seed-specific, can be distinguished [43]. Thus, among five FAD2 copies identified in soybean, $F A D 2-1 A$ and $F A D 2-1 B$ were expressed specifically in immature seeds, encoding enzymes responsible for the synthesis of seed polyunsaturated fatty acids, whereas $F A D 2-2 A, F A D 2-2 B$, and $F A D 2-2 C$ were constitutive expression and encoding enzymes responsible for membrane lipid desaturation [44]. The phylogenetic analysis showed that the AsFAD2-1 gene belonged to seed-specific expression (Fig. 1), which was consistent with its tissue expression profile (Fig. 3), whereas AsFAD2-1 was strongly expressed in developing seeds, which was similar to the expression patterns of FAD2-1 genes in most plants such as cotton and grape [33, 45]. The phylogenetic analysis also revealed that the AsFAD2-
10 gene was constitutively expressed (Fig. 1), which was consistent with the results of tissue expression (Fig. 3). The AsFAD2 genes had the highest homology with the safflower CtFAD2 genes (Fig. 1), apparently because both species belong to the same Compositae family, i.e., have close genetic relationship.

The regulation of FAD2 gene is important in understanding the composition of fatty acids and biosynthesis, plant development, and essential role in biotic and abiotic stresses [8]. Under salt stress, FAD2 enzymes play a key role in regulating and maintaining lipid composition, biophysical properties, and normal function of membrane-bound proteins [8]. In high salt-exposed Arabidopsis, the expression of FAD2 mutants resulted in low levels of PUFAs, which decreased membrane lipid fluidity and salt tolerance [19]. Two ShFAD2 genes from Salvia hispanica were differentially upregulated or repressed by salt stress [22]. In this study, except AsFAD2-1 and - 10 were downregulated, AsFAD2-2, 15 , and -22 were upregulated at $50 \mathrm{mM} \mathrm{NaCl}$; AsFAD2-2 and -5 were increased and AsFAD2-7 was decreased significantly at $200 \mathrm{mM} \mathrm{NaCl}$ compared to control. Other genes showed no difference compared to control under salt stress. The total expression level of eleven AsFAD2 genes was not affected (Fig. 4). Our previous study has shown that $A$. sphaerocephala could maintain its membrane unsaturation degree at a relatively stable level under salt stress [27]. In this research, the completely opposite response of the different member of $A s F A D 2$ family indicates that AsFAD2 family could help the plant to maintain the balance of oleic acid 


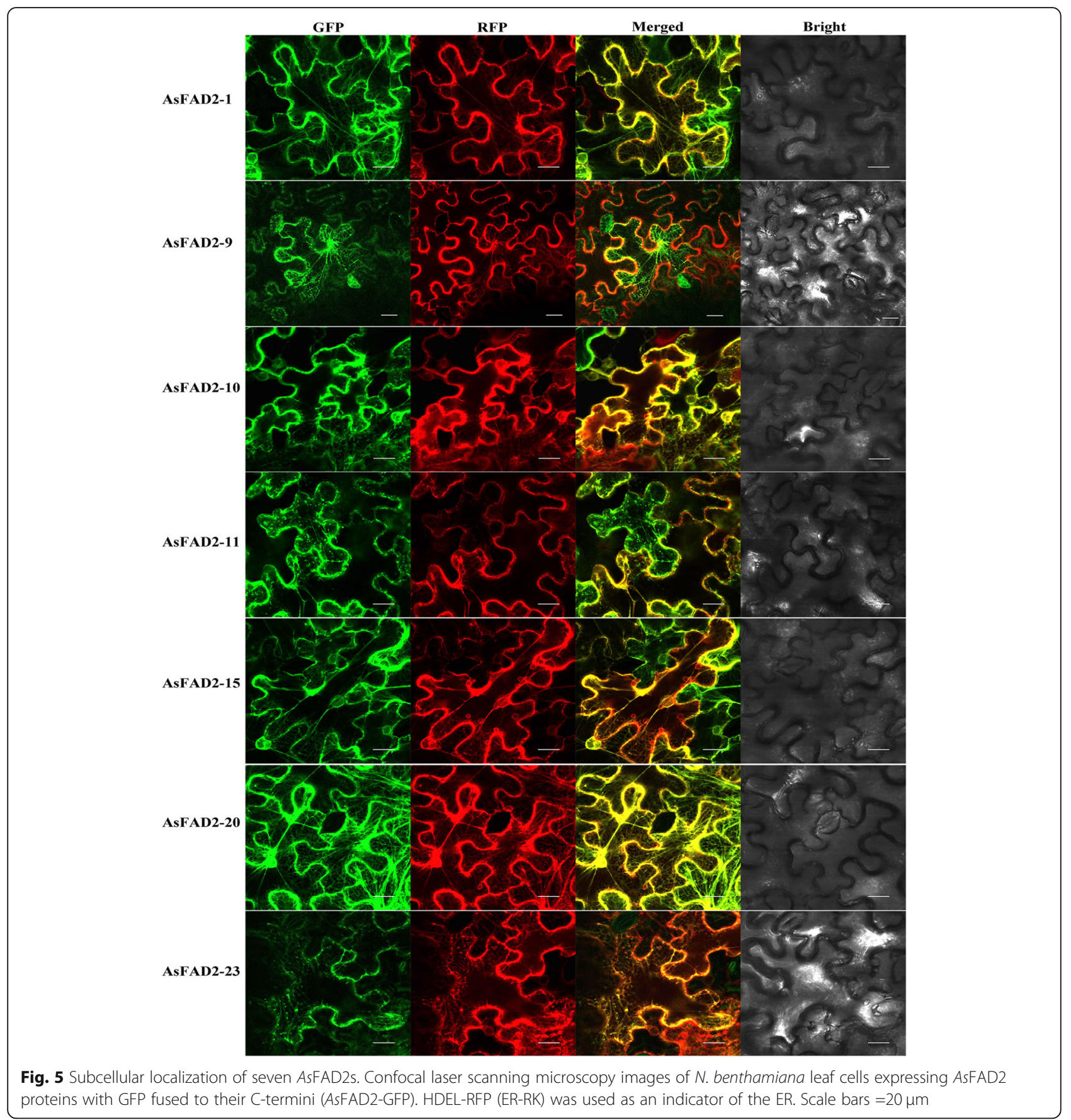

and linoleic acid under salt stress, which has not been reported before. Overall, these results suggested that the $A s F A D 2$ gene family members might adjust to appropriate levels to protect the cell membrane of $A$. sphaerocephala from salt stress. The relationship between the responses of these AsFAD2 genes to various stresses and fatty acid composition of the plant need further study.

Previous study suggested that fatty acid modifications, including elongation and desaturation, occur on the ER membrane [46]. Seven AsFAD2 proteins were located in the ER (Fig. 5), which was in agreement with previous findings for cotton FAD2-4:GFP [47], three BnFAD2s: YFP [13] and FrFAD2-1:GFP [48]. The above results were also consistent with the prediction of subcellular localization by Plant-mPLoc 2.0 (Additional file 1 : Table S1). We deduced the other AsFAD2 enzymes may be also ER-localized. These results further confirmed that the core reaction of LA biosynthesis in plants occurs in the ER. In addition, the fatty acid composition of $N$. benthamiana leaves expressing seven AsFAD2s were 


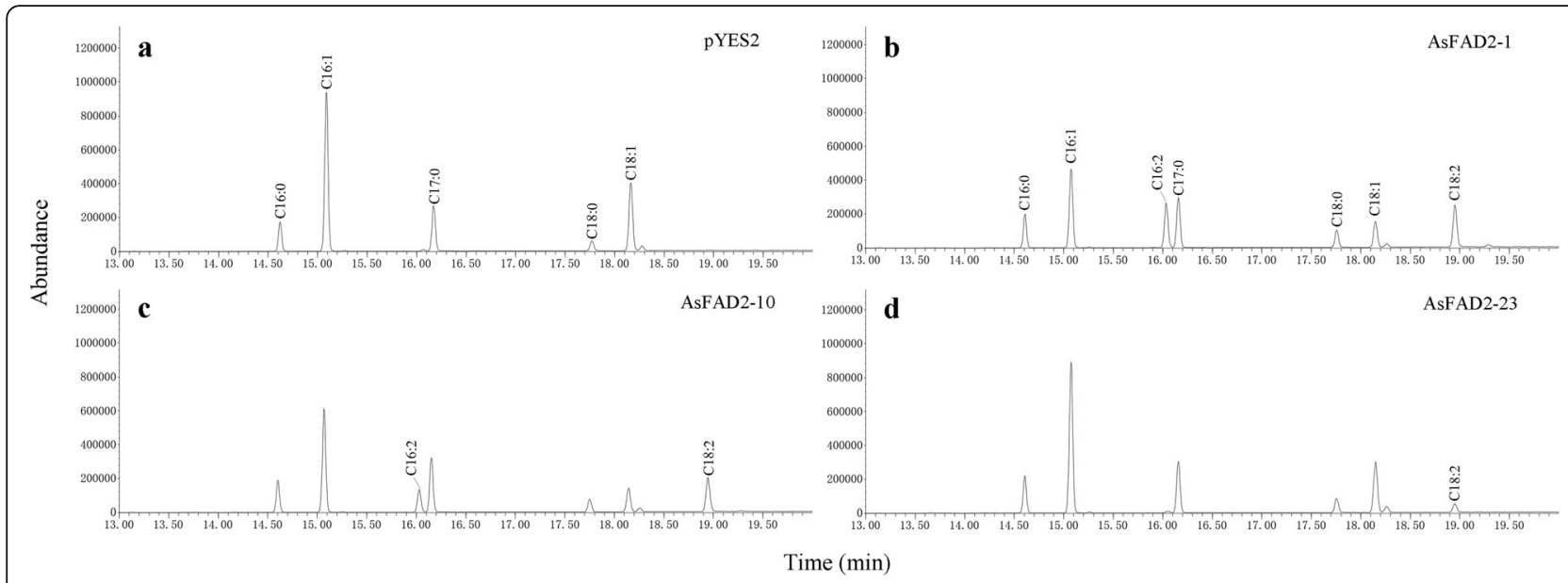

Fig. 6 GC-MS of yeast cultures transformed with the pYES2 vector alone (a), pYES2 with AsFAD2-1 (b), pYES2 with AsFAD2-10 (c), pYES2 with AsFAD2-23 (d). C16:0, palmitic acid; C16:1, palmitoleic acid; C16:2, palmitolinoleic acid; C18:0, stearic acid; C18:1, oleic acid; C18:2, linoleic acid; C17:0 as internal standard

detected, and no additional novel fatty acids was found (such as crepenynic acid) compared to the controls (data not presented). This result was different from the CtFAD2-11 [15].

S. cerevisiae INVSc1 is a suitable heterologous expression system for functional studies of FAD2 enzymes, because it has a simple fatty acid profile, contains the FAD2 substrate (oleic acid), and lacks endogenous FAD2 activity. Functional analysis of FAD2 enzymes from many plants such as A. thaliana [49], Tung [50], Soybean [45], and Camelina sativa [51] were successfully performed in yeast, where recombinant enzymes produced certain amounts of LA. In this study, sixteen AsFAD2 genes were expressed in S. cerevisiae INVSc1, which were then analyzed for fatty acid composition. It was found that AsFAD2-1, - 10, 23 could effect the conversion of $\mathrm{C} 18: 1$ to $\mathrm{C} 18: 2$ in transgenic yeast, whereas no C18:2 was detected in the controls. Furthermore, AsFAD2-1 and AsFAD2-10 could also convert C16:1 to C16:2. These results indicated that AsFAD2-1 and $A s$ FAD2-10 were both $\Delta^{12}$ oleate desaturases and $\Delta^{12}$ palmitoleate desaturase. Previous study showed that C16:2 was generated in the plastid of plant by FAD6 activity [52]. This study showed that C16:2 could also be produced by FAD2 in the ER. Similar results have been found in other studies [13, 15, 48, 51], and the reasons need further study. In this study, the corresponding fatty acid products were not detected in yeast cells expressing other AsFAD2 genes. Similar results were obtained in safflower, where five CtFAD2 family members were found to be functional and six non-functional [15]. Although the heterologous expression system in yeast is normally used to study the function of plant PUFA biosynthesis enzymes, numerous factors still mediate the enzyme activity, such as yeast strain, promoter type, and culture condition [1]. In addition, we speculated neofunctionization, pseudogenization could also cause those genes had no functions in yeast, although they could expressed in the tissues of $A$. sphaerocephala.

\section{Conclusions}

In this study, we cloned and characterized a large FAD2 gene family from $A$. sphaerocephala. The coding region sequences of $A s F A D 2$ gene family were highly homologous and relatively conservative during evolution. The expression of $A s F A D 2$ gene family members had temporal and spatial differences. However, the overall expression of AsFAD2 genes remained stable under salt stress. AsFAD2 proteins were all located in the ER. Three AsFAD2 enzymes were confirmed in transgenic yeasts as $\Delta^{12}$ fatty acid desaturases.

\section{Methods \\ Plant material}

We used seventeen samples of A. sphaerocephala, including seeds after 3 and 7 days of germination, seedlings, roots, stems, leaves, flower buds, flowers, early developing, mid-developing, and mature seeds, and six different callus tissues. Leaves, stems, roots, flowers, flower buds, early developing seeds, mid-developing seeds and mature seeds were collected from A. sphaerocephala plants (voucher No. 0019079, identified by Quanru Liu and deposited at Hebei Normal University, http://www.nsii.org.cn/node/79/cvh/157/2ef/15103591) growing in the Alxa Desert of Inner Mongolia, northwest China (N: $38^{\circ} 68^{\prime}$, E: $\left.105^{\circ} 61^{\prime}\right)$. No specific permission was required for use of these materials for experimental purposes. In addition, seeds after 3 and 7 days of germination, seedlings, and six different callus tissues were collected from the laboratory of Lanzhou 
University, Lanzhou, China. The collection of all samples completely complies with local and national legislation permission. These samples were taken as same as our previous work [16]. One month old Nicotiana tabacum plants were used for transient expression of AsFAD2 genes to determine the subcellular localization of the encoded FAD2 proteins. N. tabacum seeds were preserved in our lab.

Isolation of the full-length cDNA of AsFAD2 gene family.

Total RNA was extracted from each plant sample using the UNlQ-10 Column Trizol Total RNA Isolation Kit (Sangon, China) and analyzed for concentration and quality using NanoDrop ND1000 (Thermo Fisher Scientific, USA) and gel electrophoresis. Based on our previous study [16], nucleotide sequences of twenty-six AsFAD2 genes determined by RNA-seq (Additional file 7: Table S4). 5'/3' RACE gene-specific primers for each $A s F A D 2$ gene were designed by Primer 5.0 and synthesized by the Sangon Company (Additional file 8: Table S5 and Additional file 9: Table S6). Total RNA of seventeen $A$. sphaerocephala samples ( $1 \mu \mathrm{g}$ for each) was used as a template to synthesize first-strand CDNA by $5^{\prime}$ and 3' RACE, respectively, using a SMARTer RACE 5'/3' Kit (Clontech, Japan) according to the manufacturer's instructions. Finally, the full-length cDNA of each AsFAD2 gene was obtained by splicing of $5^{\prime}$ and $3^{\prime}$ sequences and reference sequences using the DNAMAN 6.0 software.

The open reading frame (ORF) of each AsFAD2 gene was identified using the online ORF finder software (https://www.ncbi.nlm.nih.gov/orffinder/). Primers were designed based on the region upstream of the start codon and downstream of the stop codon (both codons were included) (Additional file 10: Table S7) and ORFs were amplified by PCR using PrimeSTAR HS DNA Polymerase (Takara, Japan). The resultant products were purified by the TaKaRa MiniBEST Agarose Gel DNA Extraction Kit (Takara, Japan), subcloned into the pLB vector (Tiangen, China), and used to transform E. coli (Transgen, China). All constructs were verified by sequencing.

Bioinformatics analysis.

The characteristics of AsFAD2 genes were analyzed using several online resources. Nucleotide and deduced amino acid sequences were identified by NCBI BLAST (http://www.ncbi.nlm.nih.gov/BLAST/) and physicochemical properties of putative proteins predicted using ProtParam (http://web.expasy.org/protparam/). TMHMM (http://www.cbs.dtu.dk/services/TMHMM/) and PlantmPLoc $2.0 \quad$ (http://www.csbio.sjtu.edu.cn/bioinf/plantmulti/) servers were used to predict transmembrane regions and subcellular location, respectively. Sequence motifs were searched and analyzed using the MEME web server (http://meme-suite.org/tools/meme) and TBtools software. A phylogenetic tree was constructed by MEGA6.0 using the maximum likelihood method, and bootstrapping with 1000 replicates was used to establish the confidence limit of the tree branches.

Quantitative real-time PCR (qRT-PCR) analysis.

Total RNA was extracted from ten tissues of $A$. sphaerocephala (germinated seeds after 3 and 7 days, seedlings, roots, stems, leaves, flower buds, flowers, early and mid-developing seeds) using an RNA Isolation kit (Sangon, China), reverse-transcribed into cDNA using the PrimeScript RT reagent Kit With gDNA Eraser (Takara, Japan), and analyzed by qRT-PCR in an ABI 7500 thermocycler (Applied Biosystems, USA) using a SYBR Premix Ex Taq Kit (Takara, Japan). Primers for the sixteen AsFAD2 genes were presented in Additional file 11: Table S8. PCR conditions were as follows: $95^{\circ} \mathrm{C}$ for $30 \mathrm{~s}$, and 40 cycles of $95^{\circ} \mathrm{C}$ for $5 \mathrm{~s}$ and $60^{\circ} \mathrm{C}$ for $1 \mathrm{~min}$. Relative gene expression was calculated by the $2^{-\Delta \mathrm{Ct}}$ method [53] and presented as the mean $\pm \mathrm{SE}$ of three replicates, the actin-encoding gene was used as an internal control.

Subcellular localization of AsFAD2 genes in Nicotiana benthamiana leaves.

To observe the subcellular localization of AsFAD2 proteins, the coding sequences of selected seven representative AsFAD2 genes without the stop codons were respectively amplified by PCR using primers listed in Additional file 12: Table S9 and then inserted into the Xho I and Sal I sites of the pBI121-EGFP vector (Miaolingbio, China) using the In-Fusion ${ }^{\circ}$ HD Cloning Kit (Takara, Japan). So, the DNA fragments of target genes were respectively fused to the $\mathrm{N}$-terminal region of GFP under the control of the CaMV35S promoter. The recombinant vectors were named as pAsFAD2: EGFP. The pHDEL:RFP (mCherry) plasmid was used to mark the ER. The pAsFAD2:EGFP and pHDEL:RFP were independently transformed into the Agrobacterium tumefaciens GV3101. The two cultures $\left(\mathrm{OD}_{600}=0.8\right)$ were mixed (1:1) and co-infiltrated into epidermal tissues of $N$. benthamiana leaves using infiltration buffer (10 mM MES, $10 \mathrm{mM} \mathrm{MgCl} \cdot 6 \mathrm{H}_{2} \mathrm{O}, 100 \mu \mathrm{M}$ acetosyringone, $\mathrm{PH}=5.7$ ) [54]. Transfected leaves regions were examined at $48 \mathrm{~h}$ after injection, and analyzed with a confocal laser scanning microscope (FV1000 MPE, Olympus) at the excitation wave lengths of 488 and 561 $\mathrm{nm}$ to visualize GFP and RFP fluorescence, respectively.

Heterogonous expression of AsFAD2 genes in Saccharomyces cerevisiae.

The coding sequences of sixteen AsFAD2 genes were amplified using specific primers (Additional file 13: Table S10) and inserted into the shuttle vector pYES2 (Invitrogen, USA), which harbored the GAL1 promoter for inducing gene expression by galactose [47]. The resultant constructs were sequenced and introduced into 
Saccharomyces cerevisiae INVSc1 (Invitrogen, USA) using a Quick \& Easy Yeast Transformation Mix kit (Takara, Japan). Yeast colonies were selected on synthetic complete medium lacking uracil (SC-U) and containing $2 \%$ glucose $(\mathrm{w} / \mathrm{v})$, and single colonies were grown in liquid medium at $30^{\circ} \mathrm{C}$ with shaking for $24 \mathrm{~h}$. Yeast cells were harvested by centrifugation at $1500 \mathrm{~g}$ for 5 min, diluted to $\mathrm{OD}_{600}=0.4$, and induced using $\mathrm{SC}-\mathrm{U}$ liquid medium with $2 \%$ galactose and $1 \%$ raffinose at $22^{\circ} \mathrm{C}$ for 3 days. Cells were harvested by centrifugation, washed in sterile water three times, and freeze-dried in a lyophilizer.

Total fatty acids were extracted from $0.5 \mathrm{~g}$ of yeast cells, and fatty acid methyl esters were analyzed by gas chromatography (Agilent $6890 \mathrm{~N}$, USA) and mass spectrometry (Agilent 5975C) using a polar capillary column (Agilent DB-FFAP) as previously described [55]; heptadecanoic acid (C17:0) was used as internal standard.

\section{Statistical analysis}

Data were subjected to one-way analysis of variance (ANOVA) using SPSS 17.0 (SPSS Inc., Chicago, IL, USA). The significant differences among means were identified by Duncan's multiple range tests at a significance level of $P<0.05$. Data were presented as means \pm $\mathrm{SE}(n=3)$.

\section{Supplementary information}

Supplementary information accompanies this paper at https://doi.org/10. 1186/s12870-019-2083-5.

Additional file 1: Table S1. Analysis of the full-length CDNA sequences of AsFAD2 gene family and its predicted amino acid sequence characteristics.

Additional file 2: Figure S1. Sequence similarity of the coding region deduced amino acids of twenty-one ASFAD2 genes.

Additional file 3: Table S2. Sixteen AsFAD2 putative polypeptide sequences from A. sphaerocephala.

Additional file 4: Figure S2. The alignment of the putative polypeptide sequences of AsFAD2 genes together with those of selected ortholog plants.

Additional file 5: Figure S3. The detailed information of putative twenty conserved motifs.

Additional file 6: Table S3. Fatty acid composition of transgenic yeast cells.

Additional file 7: Table S4. Nucleotide sequences of twenty-six FAD2 unigenes from $A$. sphaerocephala transcriptome.

Additional file 8: Table S5. Primers used in the 5'RACE of twenty-one AsFAD2 genes in A. sphaerocephala.

Additional file 9: Table S6. Primers used in the 3'RACE of twenty-one AsFAD2 genes in A. sphaerocephala.

Additional file 10: Table S7. Primers used for amplification of the ORF of sixteen AsFAD2 genes in A. sphaerocephala.

Additional file 11: Table S8. Primers used for qRT-PCR study of sixteen AsFAD2 genes in A. sphaerocephala.

Additional file 12: Table S9. Primers used in subcellular localization study of seven AsFAD2 genes.
Additional file 13: Table S10. Primers carrying restriction

endonuclease used in heterologous expression of sixteen AsFAD2 genes in Saccharomy cescerevisiae.

\section{Abbreviations}

AA: Amino acid; CDNA: Complementary DNA; CLA: Conjugated linoleic acid; FAD2: Microsomal delta-12 $\left(\Delta^{12}\right)$ oleate desaturase; GFP: Green fluorescent protein; GRAVY: Grand average of hydropathicity; MW: Molecular weight; NCBI: National Center for Biotechnology Information; ORF: Open reading frame; PCR: Polymerase chain reaction; PI: Isoelectric point;

PUFA: Polyunsaturated fatty acid; qRT-PCR: Quantitative real-time PCR; RACE: Rapid Amplification of cDNA Ends; RFP: Red Fluorescent protein; RTPCR: Reverse transcription PCR; UTR: Untranslated region

\section{Acknowledgments}

Not applicable.

\section{Authors' contributions}

$\mathrm{LZ}, \mathrm{HF}$ conceived the project and designed experiments; XM, XH, SN, XC performed experiments; $X M$ and $L Z$ analyzed experiment data and wrote the manuscript. All authors read and approved the final manuscript.

\section{Funding}

This work was supported by the National Basic Research Program of China (2014CB138703); National Natural Science Foundation of China (31770763): National Key R\&D Program of China (2016YFC0500506); The Fundamental Research Funds for the Central Universities (Izujbky-2017-54); The Changjiang Scholars and Innovative Research Team in University (IRT_17R50); The 111 project (B12002). These funding bodies were not involved in the design of the study and collection, analysis, and interpretation of data and in writing the manuscript.

\section{Availability of data and materials}

All the data pertaining to the present study have been included in the tables and figures of the manuscript, and the authors are pleased to share all the data and plant materials upon reasonable request.

\section{Ethics approval and consent to participate}

This study has not directly involved humans or animals. The leaves, stems, roots, flowers, flower buds, early developing seeds, mid-developing seeds and mature seeds were collected from A. sphaerocephala plants growing in the Alxa Desert of Inner Mongolia, northwest China (N: $38^{\circ} 68^{\prime}$, E: $105^{\circ} 61^{\prime}$ ). No specific permission was required for use of these materials for experimental purposes. The seeds after 3 and 7 days of germination, seedlings, and six different callus tissues were collected from the laboratory of Lanzhou University, Lanzhou, China as per standard practices, and samples were harvested at the required time. We comply with the Convention on the Trade in Endangered Species of Wild Fauna and Flora.

Consent for publication

Not applicable.

\section{Competing interests}

The authors declare that they have no conflict of interest.

Received: 29 May 2019 Accepted: 17 October 2019

Published online: 08 November 2019

\section{References}

1. Chodok P, Eiamsa-Ard P, Cove DJ, Quatrano RS, Kaewsuwan S. Identification and functional characterization of two $\Delta(12)$-fatty acid desaturases associated with essential linoleic acid biosynthesis in Physcomitrella patens. J Ind Microbiol Biotechnol. 2013;40(8):901-13.

2. Pereira SL, Leonard AE, Mukerji P. Recent advances in the study of fatty acid desaturases from animals and lower eukaryotes. Prostag Leukotr Ess. 2003; 68(2):97-106.

3. Zhang HT, Shan $L$, Quan $X Q$, BI Y p, Yang JS, Wang XL. Functional analysis of an Arachis hypogaea L. $\Delta 12$ fatty acid Desaturase gene by heterologous expression in Saccharomyces cerevisiae. J Peanut Sci. 2006;35(1):1-7. 
4. Dilzer A, Park Y. Implication of conjugated linoleic acid (CLA) in human health. Crit Rev Food Sci Nutr. 2012;52(6):488-513.

5. Tanaka K. Occurrence of conjugated linoleic acid in ruminant products and its physiological functions. Anim Sci J. 2005;76(4):291-303.

6. Warude D, Joshi K, Harsulkar A. Polyunsaturated fatty acids: biotechnology. Crit Rev Biotechnol. 2006;26(2):83-93.

7. Upchurch RG. Fatty acid unsaturation, mobilization, and regulation in the response of plants to stress. Biotechnol Lett. 2008;30(6):967-77.

8. Dar AA, Choudhury AR, Kancharla PK, Arumugam N. The FAD2 gene in plants: occurrence, regulation, and role. Front Plant Sci. 2017:8:1789.

9. Shanklin J, Cahoon EB. Desaturation and related modifications of fatty acids. Annu Rev Plant Physiol Plant Mol Biol. 1998;49(49):611-41.

10. Malcicka M, Visser B, Ellers J. An evolutionary perspective on linoleic acid synthesis in animals. Evol Biol. 2018;45(1):15-26.

11. Okuley J, Lightner J, Feldmann K, Yadav N, Lark E, Browse J. Arabidopsis FAD2 gene encodes the enzyme that is essential for polyunsaturated lipid synthesis. Plant Cell. 1994;6(1):147-58.

12. Hernández ML, Mancha M, Martínez-Rivas JM. Molecular cloning and characterization of genes encoding two microsomal oleate desaturases (FAD2) from olive. Phytochemistry. 2005;66(12):1417-26.

13. Lee KR, Sohn SI, Jin HJ, Sun HK, Roh KH, Kim JB, Mi CS, Kim HU. Functional analysis and tissue-differential expression of four FAD2 genes in amphidiploid Brassica napus derived from Brassica rapa and Brassica oleracea. Gene. 2013;531(2):253-62.

14. Wang $Y$, Zhang $X$, Zhao $Y$, Prakash CS, He G, Yin D. Insights into the novel members of the FAD2 gene family involved in high-oleate fluxes in peanut. Genome. 2015;58(8):375-83.

15. Cao SJ, Zhou XR, Wood CC, Green AG, Singh SP, Liu L, Liu Q. A large and functionally diverse family of Fad2 genes in safflower (Carthamus tinctorius L.). BMC Plant Biol. 2013;13(1):5.

16. Zhang L, Hu X, Miao X, Chen X, Nan S, Hua F. Genome-scale Transcriptome analysis of the desert shrub Artemisia sphaerocephala. PLoS One. 2016;11(4): e0154300.

17. Guo YL. Gene family evolution in green plants with emphasis on the origination and evolution of Arabidopsis thaliana genes. Plant J. 2013;73(6): 941-51

18. Miquel M, James D, Dooner $\mathrm{H}$, Browse J. Arabidopsis requires polyunsaturated lipids for low-temperature survival. Proc Natl Acad Sci U S A. 1993;90(13):6208-12

19. Zhang J, Liu H, Sun J, Li B, Zhu Q, Chen S, Zhang H. Arabidopsis fatty acid Desaturase FAD2 is required for salt tolerance during seed germination and early seedling growth. PLoS One. 2012;7(1):e30355.

20. Kargiotidou A, Deli D, Galanopoulou D, Tsaftaris A, Farmaki T. Low temperature and light regulate delta 12 fatty acid desaturases (FAD2) at a transcriptional level in cotton (Gossypium hirsutum). J Exp Bot. 2008;59(8): 2043-56.

21. Rodríguez-Vargas S, Sánchez-García A, Martínez-Rivas JM, Prieto JA, RandezGil F. Fluidization of membrane lipids enhances the tolerance of Saccharomyces cerevisiae to freezing and salt stress. Appl Environ Microbiol. 2007;73(1):110-6.

22. Xue $Y$, Yin N, Chen B, Liao F, Win AN, Jiang J, Wang R, Jin X, Lin N, Chai Y. Molecular cloning and expression analysis of two FAD2 genes from chia (Salvia hispanica). Acta Physiologiae Plantarum. 2017:39-95(4):95.

23. Board E. Flora of China. Beijing: Science Press 1991;76(2):189.

24. Zhang J, Ma J, Yao J, Bai Z, Yang Y, Huang A, Du J. Study on exploitation and utilization on wild Artemisia sphaerocephala. Pratacultural Sci. 2002; 19(7):10-3.

25. Fu H, Wang J, Wang Z, Chen L. Fatty acid and amino acid compositions of Artemisia sphaerocephala seed and its influence on mouse hyperlipidemia. Chem Nat Compd. 2011;47(4):675-8.

26. Miao XM, Zhang $\sqcup$, Chen XL, Wu SJ, Niu DC, Fu H. The relationship of fatty acid composition and resistance of Artemisia shaerocephala seedlings under water stress. Acta Pratacul Sin. 2015;24(2):55-61.

27. Chen XL, Zhang LJ, Miao XM, Hu XW, Nan SZ, Wang J, Fu H. Effect of salt stress on fatty acid and a-tocopherol metabolism in two desert shrub species. Planta. 2017;247(2):499-511.

28. Schmutz J, Cannon SB, Schlueter J, Ma JX, Mitros T, Nelson W, Hyten DL, Song QS, Thelen JJ, XD CJ, Hellsten U, May GD, Yu Y, Sakurai T, Umezawa T, Bhattacharyya MK, Sandhu D, Valliyodan B, Lindquist E, Peto M, Grant D, Shu S, Goodstein D, Barry K, Futrell-Griggs M, Abernathy B, Du J, Tian Z, Zhu L, Gill N, Joshi T, Libault M, Sethuraman A, Zhang XC, Shinozaki K, Nguyen HT,
Wing RA, Cregan P, Specht J, Grimwood J, Rokhsar D, Stacey G, Shoemaker $\mathrm{RC}$, Jackson SA. Genome sequence of the palaeopolyploid soybean. Nature. 2010;463(7278):178-83.

29. Lakhssassi N, Zhou Z, Liu S, Colantonio V, Abughazaleh A, Meksem K. Characterization of the FAD2 gene family in soybean reveals the limitations of gel-based TILLING in genes with high copy number. Front Plant Sci. 2017;8:324.

30. Dong CJ, Cao N, Zhang ZG, Shang QM. Characterization of the fatty acid Desaturase genes in cucumber: structure, phylogeny, and expression patterns. PLoS One. 2016;11(3):e0149917.

31. Tai LH, Wang TJ. Odonhuar, Sainjirgal, Altanbagan. Study on the karyotype of six Sandy subshrub species of Artemisia L. J Inner Mongolia Norm Univ. 2012;41(4):420-7.

32. Minto RE, Gibbons WJ Jr, Cardon TB, Lorigan GA. Synthesis and conformational studies of a transmembrane domain from a diverged microsomal Delta(12)-desaturase. Anal Biochem. 2002;308(1):134-40.

33. Lee KR, Sun HK, Go YS, Jung SM, Roh KH, Kim JB, Suh MC, Lee S, Kim HU. Molecular cloning and functional analysis of two FAD2 genes from American grape ( Vitis labrusca L.). Gene. 2012;509(2):189-94.

34. Nayeri FD, Yarizade K. Bioinformatics study of delta-12 fatty acid desaturase 2 (FAD2) gene in oilseeds. Mol Biol Rep. 2014;41(8):5077-87.

35. Zhou XR, Singh SP, Green AG. Characterisation of the FAD2 gene family from Hiptage benghalensis: a ricinoleic acid accumulating plant. Phytochemistry. 2013;92(4):42-8.

36. Zhou XR, Singh S, Liu Q, Green A. Combined transgenic expression of $\Delta 12$ desaturase and $\Delta 12$-epoxygenase in high linoleic acid seeds leads to increased accumulation of vernolic acid. Funct Plant Biol. 2006;33(6):585-92.

37. Nam JW, Kappock TJ. Cloning and transcriptional analysis of Crepis alpina fatty acid desaturases affecting the biosynthesis of crepenynic acid. J Exp Bot. 2007;58(6):1421-32.

38. Okada S, Zhou XR, Damcevski K, Gibb N, Wood C, Hamberg M, Haritos VS Diversity of $\Delta 12$ fatty acid desaturases in santalaceae and their role in production of seed oil acetylenic fatty acids. J Biol Chem. 2013;288(45): 32405-13.

39. Rawat R, Yu XH, Sweet M, Shanklin J. Conjugated fatty acid synthesis: residues 111 and 115 influence product partitioning of Momordica charantia conjugase. J Biol Chem. 2012;287(20):16230-7.

40. Yurchenko O, Shockey JM, Gidda SK, Silver MI, Chapman KD, Mullen RT, Dyer JM. Engineering the production of conjugated fatty acids in Arabidopsis thaliana leaves. Plant Biotechnol J. 2017;15(8):1010-23.

41. Broun P, Boddupalli S, Somerville C. A bifunctional oleate 12-hydroxylase: desaturase from Lesquerella fendleri. Plant J. 1998;13(2):201-10.

42. Carlsson AS, Thomaeus S, Hamberg M, Stymne S. Properties of two multifunctional plant fatty acid acetylenase/desaturase enzymes. Eur J Biochem. 2004;271(14):2991-7.

43. Martínez-Rivas JM, Sperling P, Lühs W, Heinz E. Spatial and temporal regulation of three different microsomal oleate desaturase genes (FAD2) from normal-type and high-oleic varieties of sunflower (Helianthus annuus L. ). Mol Breed. 2001;8(2):159-68.

44. Schlueter JA, Vasylenkosanders IF, Deshpande S, Yi J, Siegfried M, Roe BA, Schlueter SD, Scheffler BE, Shoemaker RC. The FAD2 gene family of soybean: insights into the structural and functional divergence of a paleopolyploid genome. Crop Sci. 2007:47(Supplement_1):14-26.

45. Li L, Wang X, Gai J, Yu D. Molecular cloning and characterization of a novel microsomal oleate desaturase gene from soybean. J Plant Physiol. 2007; 164(11):1516-26.

46. Jung JH, Kim H, Go YS, Lee SB, Hur CG, Kim HU, Suh MC. Identification of functional BrFAD2-1 gene encoding microsomal delta-12 fatty acid desaturase from Brassica rapa and development of Brassica napus containing high oleic acid contents. Plant Cell Rep. 2011;30(10):1881-92.

47. Zhang DY, Pirtle IL, Park SJ, Nampaisansuk M, Neogi P, Wanjie SW, Pirtle RM, Chapman KD, Lessire R, Bessoule JJ. Identification and expression of a new delta-12 fatty acid desaturase (FAD2-4) gene in upland cotton and its functional expression in yeast and Arabidopsis thaliana plants. Plant Physiol Biochem. 2009;47(6):462-71.

48. Lee KR, Lee $Y$, Kim EH, Lee SB, Roh KH, Kim JB, Kang HC, Kim HU. Functional identification of oleate 12-desaturase and $\omega$-3 fatty acid desaturase genes from Perilla frutescens var. frutescens. Plant Cell Rep. 2016;35(12):2523-37.

49. Covello PS, Reed DW. Functional expression of the extraplastidial Arabidopsis thaliana oleate desaturase gene (FAD2) in Saccharomyces cerevisiae. Plant Physiol. 1996;111(1):223-6. 
50. Dyer JM, Mullen RT, Mckeon TA. Molecular analysis of a Bifunctional fatty acid Conjugase/Desaturase from Tung. Implications for the evolution of plant fatty acid diversity. Plant Physiol. 2002;130(4):2027-38.

51. Rodríguez-Rodríguez MF, Salas JJ, Venegas-Calerón M, Garcés R, MartínezForce E. Molecular cloning and characterization of the genes encoding a microsomal oleate $\Delta^{12}$ desaturase (CSFAD2) and linoleate $\Delta^{15}$ desaturase (CSFAD3) from Camelina sativa. Ind Crop Prod. 2016;89:405-15.

52. Wallis JG, Browse J. Mutants of Arabidopsis reveal many roles for membrane lipids. Prog Lipid Res. 2002;41(3):254-78.

53. Schmittgen TD, Livak KJ. Analyzing real-time PCR data by the comparative CT method. Nat Protoc. 2008;3(6):1101-8.

54. Wydro M, Kozubek E, Lehmann P. Optimization of transient Agrobacteriummediated gene expression system in leaves of Nicotiana benthamiana. Acta Biochim Pol. 2006;53(2):289-98.

55. Mao ZX, Fu H, Nan ZB, Wang J, Wan CG. Fatty acid content of common vetch (Vicia sativa L.) in different regions of Northwest China. Biochem Syst Ecol. 2012;44(44):347-51.

\section{Publisher's Note}

Springer Nature remains neutral with regard to jurisdictional claims in published maps and institutional affiliations.

Ready to submit your research? Choose BMC and benefit from:

- fast, convenient online submission

- thorough peer review by experienced researchers in your field

- rapid publication on acceptance

- support for research data, including large and complex data types

- gold Open Access which fosters wider collaboration and increased citations

- maximum visibility for your research: over $100 \mathrm{M}$ website views per year

At $\mathrm{BMC}$, research is always in progress.

Learn more biomedcentral.com/submissions 